\title{
5-Gbit/s BER Performance on an All Fiber-Optic Add/Drop Device Based on a Taper-Resonator-Taper Structure
}

\author{
Ming Cai, Per Olof Hedekvist, Ashish Bhardwaj, and Kerry Vahala, Member, IEEE
}

\begin{abstract}
We present an all fiber-optic add/drop device based on a taper-resonator-taper structure with improved characteristics. Several gigahertz bandwidths are observed using microspheres having diameters ranging from 30 to $50 \mu \mathrm{m}$. Extinction ratios as high as $26 \mathrm{~dB}$ of the dropped channel are obtained due to nearly ideal coupling and phase matching between the fiber tapers and the small resonator. This is the first time that bit-error rate (BER) measurements have been performed on such couplers. For a device with an optical bandwidth of $3.8 \mathrm{GHz}$, the BER shows less than 2-dB penalty at $5 \mathrm{Gbit} / \mathrm{s}$ and no signs of an error floor.
\end{abstract}

Index Terms-Add/drop filter, bit-error rate, data transmission, fiber taper, microsphere resonator.

\section{INTRODUCTION}

$\mathbf{F}$ USED-SILICA microspheres exhibiting whispering gallery modes have been attracting considerable attention because of their unique properties of high $Q$ (up to $10^{10}$ [1]) and small mode volume. Applications as diverse as cavity QED [1], [2], microlasers [3], and building blocks in optical signal processing systems [4]-[6] have been proposed and investigated. We recently proposed and demonstrated a taper-resonator-taper (TRT) structure, based on fused-silica microsphere to fiber-taper coupling, as a possible add/drop device for use in wavelength-division multiplexed (WDM) systems [5]. This device exhibited high extinction ratio $(>20$ $\mathrm{dB})$, low insertion loss ( $\sim 3 \mathrm{~dB}$, dominated by taper loss $)$ as well as intrinsic fiber-optic compatibility. However, it suffered from having a narrow optical passband $(<100 \mathrm{MHz})$ and a small mode spacing $(\sim 1 \mathrm{GHz})$. Resonators of reduced dimensions and higher eccentricity than those reported in [5] are required to overcome these shortcomings.

In this letter, we present experimental results, including bit-error rate (BER) measurements on improved TRT couplers that were fabricated using reduced diameter microspheres with large optical pass bandwidths. This is to our knowledge the first report of BER performance on a resonator-based add/drop device. Resonances with several gigahertz linewidths are observed using microspheres having diameters ranging from 30 to $50 \mu \mathrm{m}$. As high as $26-\mathrm{dB}$ extinction is realized in a $45-\mu \mathrm{m}$

Manuscript received April 17, 2000; revised May 30, 2000. This work was supported by the Defense Advanced Research Projects Agency (DARPA), the Office of Naval Research (ONR), and the David Lee Center. The work of P. O. Hedekvist was supported in part by the Swedish Foundation for International Cooperation in Research and Higher Education (STINT).

The authors are with the Department of Applied Physics, California Institute of Technology, Pasadena, CA 91125 USA (e-mail: vahala@its.caltech.edu).

Publisher Item Identifier S 1041-1135(00)07463-2.

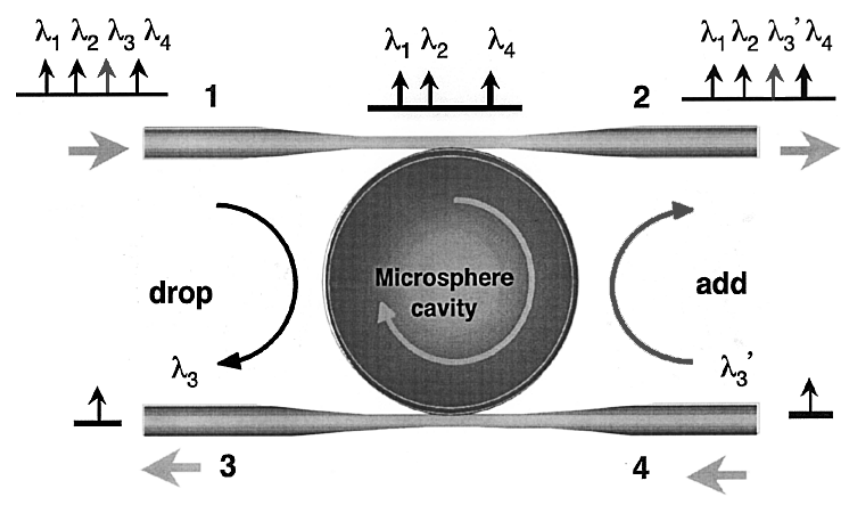

Fig. 1. Illustration of the TRT coupler. In the schematic, a channel at wavelength $\lambda_{3}$ is shown being dropped at port 3 by resonant coupling to the microsphere cavity. Another signal at wavelength $\lambda_{3}^{\prime}$ is shown being added from port 4.

diameter sphere having a resonance linewidth of $3.8 \mathrm{GHz}$. These improved devices are tested by dropping pseudorandom data streams at data rates as high as $5 \mathrm{Gbit} / \mathrm{s}$. For the device containing the $45-\mu \mathrm{m}$ microsphere, the BER curves at $5 \mathrm{Gbit} / \mathrm{s}$ exhibit less than 2-dB power penalty, whereas no penalty is observed for rates below $2.5 \mathrm{Gbit} / \mathrm{s}$.

\section{EXPERIMENTS AND RESULTS}

Fig. 1 shows a typical add/drop device geometry using a TRT coupler. A microsphere is tangentially positioned between two nearly identical tapered fibers. One fiber serves as an input port which carries a WDM signal stream containing a series of wavelength channels, $\lambda_{1}, \lambda_{2}, \lambda_{3}, \lambda_{4}, \ldots$, while the other fiber enables signal adding and dropping simultaneously. A channel at a resonant wavelength ( $\lambda_{3}$ in the figure) will be transferred to port 3 of the lower fiber via the microsphere, while other nonresonant channels will transmit through the upper fiber to port 2 with minimal power loss. Similarly, a signal $\left(\lambda_{3}^{\prime}\right)$ at the same wavelength as $\lambda_{3}$ that is input at port 4 will join the other channels at port 2 by resonant coupling.

Magnified photographs of both the top and side views of the actual system are shown in Fig. 2. Microspheres and fiber tapers under study were prepared from standard single-mode fibers. The microspheres were created by heating the tip of a tapered fiber filament using a $\mathrm{CO}_{2}$ laser. The residual stem attached to the sphere functioned as a handling rod for sphere positioning. Microspheres with diameters ranging from 30 to $50 \mu \mathrm{m}$ were fabricated in this way. Fiber tapers were pulled using a technique described in [5] and [7]. The diameters of the fiber tapers 

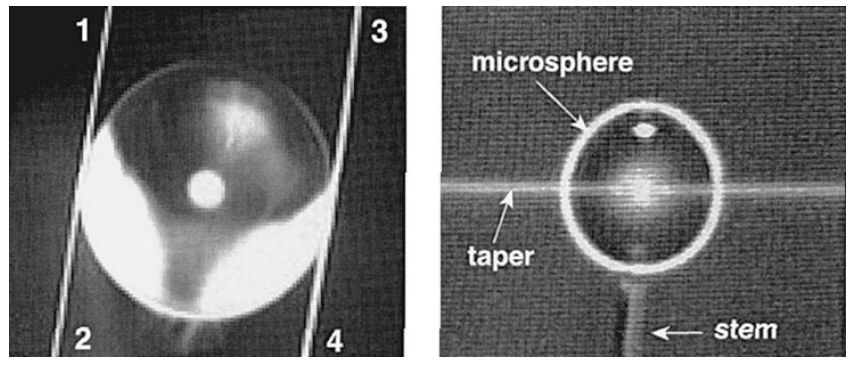

Fig. 2. Magnified pictures of the actual device both from the top and side views.

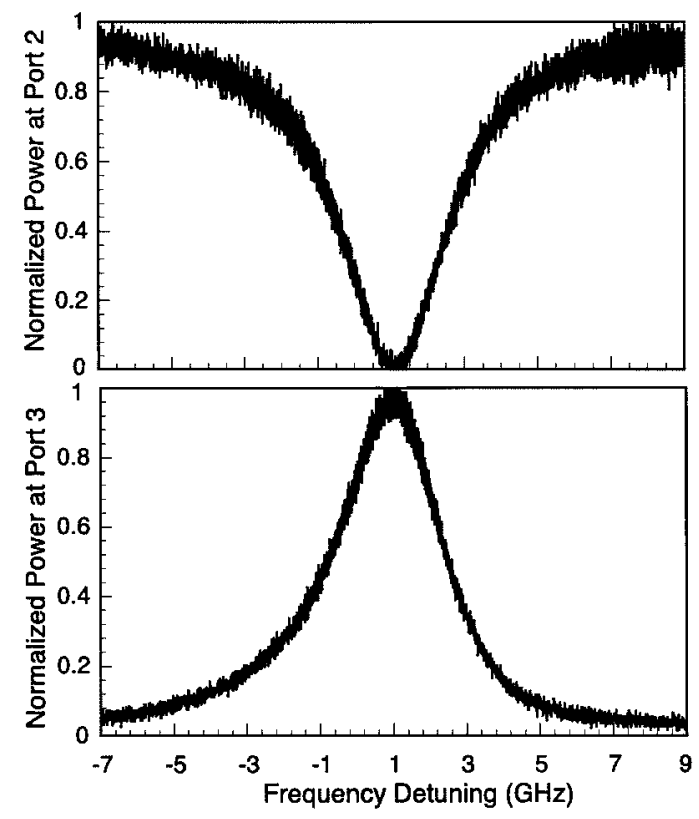

Fig. 3. Transmission spectra for the TRT coupler using a microsphere $45-\mu \mathrm{m}$ in diameter.

were tailored carefully in order to provide nearly ideal coupling and phase matching [8] between the fundamental modes in the microsphere and the tapers. The optimal taper diameters in this work were approximately $2-3 \mu \mathrm{m}$.

Transmission spectra within the $1.5-\mu \mathrm{m}$ band were measured at ports 2 and 3 simultaneously for these TRT coupling systems using reduced-size microspheres. The optical source was a New Focus tunable external-cavity diode laser having a linewidth less than $300 \mathrm{kHz}$ and a continuous-frequency tuning range of $30 \mathrm{GHz}$. Fig. 3 shows the transmission spectra of the TRT structure using the $45-\mu \mathrm{m}$ diameter microsphere. The bandwidth (FWHM) of the resonance was about $3.8 \mathrm{GHz}$. The mode spacing of two adjacent modes was measured to be about $30 \mathrm{GHz}(\sim 0.25 \mathrm{~nm})$ by coarse tuning the laser wavelength (with a resolution of $0.01 \mathrm{~nm}$ ). As high as $26-\mathrm{dB}$ extinction ratio (defined as the ratio of the transmitted optical power at the resonant wavelength to that at a nonresonant wavelength at port 2) was obtained. We believe that higher extinction levels may be possible [8] using better matched fiber tapers. The insertion loss of the dropped signal from port 1 to port

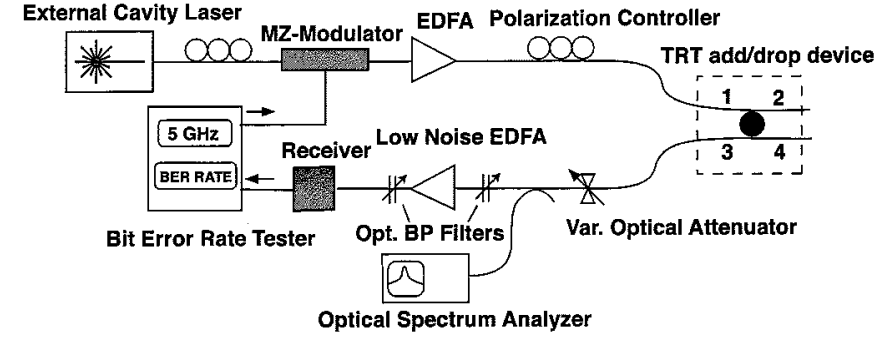

Fig. 4. BER experimental setup.

3 was approximately $3.2 \mathrm{~dB}$, and the loss of the nonresonant optical power from port 1 to port 2 was approximately $3.1 \mathrm{~dB}$. The taper loss for each coupler was measured at about $3.0 \mathrm{~dB}$ when the sphere was detached from the couplers. It is therefore reasonable to assume that the insertion losses of the device were dominated by the taper losses, which are believed to result from the sharp transition between the fiber-guided mode and the cladding-guided mode in the taper region. It is possible to reduce taper loss to as low as $0.1 \mathrm{~dB}$ by using an improved fiber taper fabrication technique as described in [9].

We evaluated the quality of the dropped signal at port 3 for data transmission purposes. BER measurements at different bit rates were performed for the couplers using different microspheres. Fig. 4 shows the experimental setup. The external cavity laser source was tuned to the central wavelength of a resonance to give the maximum power transfer from port 1 to port 3. The laser power was modulated with a $2^{15}-1$ bit PRBS from the BER tester using an external Mach-Zehnder modulator and then boosted by an erbium-doped fiber amplifier (EDFA) before launching into port 1 of the TRT coupler. The receiver consisted of a low-noise EDFA preamplifier, optical bandpass filters and a high-speed photodetector. Fig. 5 shows the BER curves obtained for the dropped signal resonantly coupled from port 1 to port 3 through the $45-\mu \mathrm{m}$ microsphere. The power penalty was less than $2 \mathrm{~dB}$ at a bit rate of $5 \mathrm{Gbit} / \mathrm{s}$. No penalties were observed at a modulation rate of $2.5 \mathrm{Gbit} / \mathrm{s}$ or below. There was also no sign of an error-rate floor at either bit rate. The eye-patterns at both bit rates are also shown as insets in Fig. 5. The experimental results obtained using other sizes of microspheres exhibited similar BER performance except that the maximum bit rates varied depending on the exact resonance bandwidth.

\section{CONCLUSION}

In consideration of practical application to WDM systems, the all-fiber microsphere-resonator-based add/drop devices have shown promising characteristics including high extinction ratio $(>26 \mathrm{~dB})$, low insertion loss $(<3 \mathrm{~dB})$, appropriate channel bandwidth (several gigahertz), fiber-optic compatibility, and compact dimensions. Additionally, BER performance is satisfactory at OC48 rates.

There still remain significant technical challenges, however, before these devices can be considered seriously for applications. The adjacent resonant mode spacing should be increased substantially. Several-terahertz separation would be desirable 


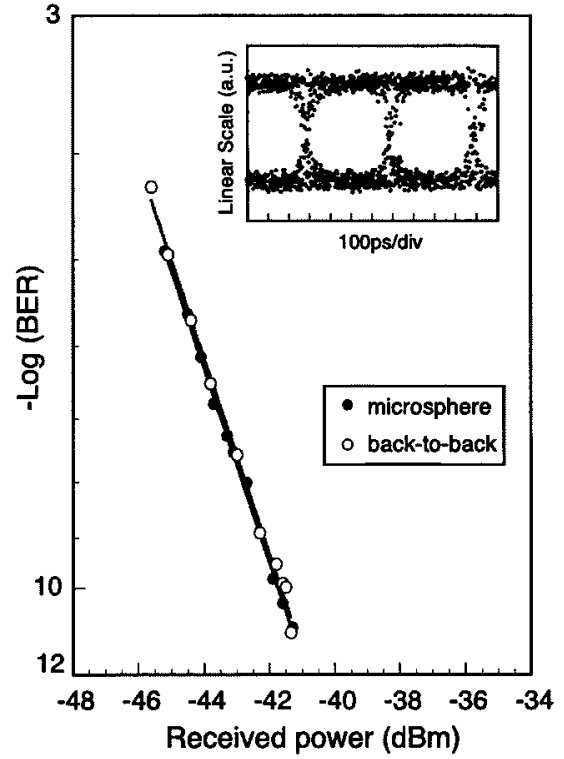

(a)

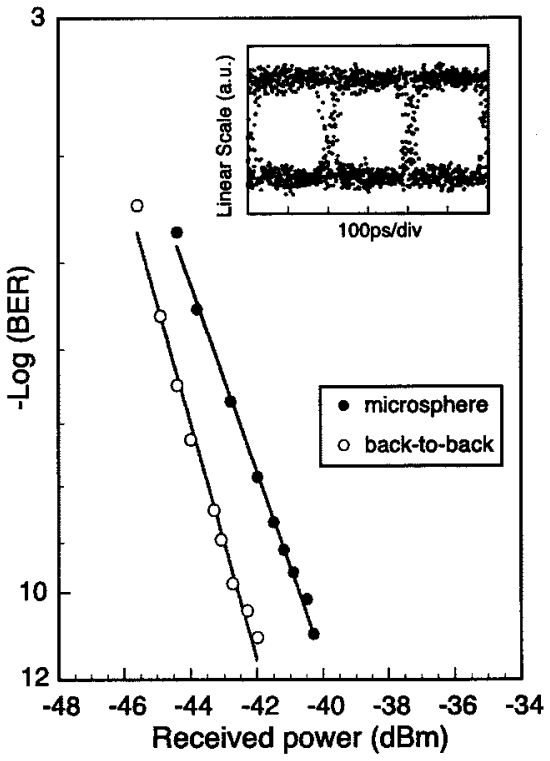

(b)

Fig. 5. BER performance of a TRT coupler using a $45 \mu \mathrm{m}$ diameter microsphere at (a) $2.5 \mathrm{Gbit} / \mathrm{s}$ and (b) $5 \mathrm{Gbit} / \mathrm{s}$, respectively. The eye patterns in both cases are shown as insets.

to enable single-channel selection by one device. Furthermore, wavelength tunability, stability, and polarization dependence are all interesting topics for future work.

\section{REFERENCES}

[1] M. L. Gorodetsky, A. A. Savchenkov, and V. S. Ilchenko, "Ultimate $Q$ of optical microsphere resonators," Opt. Lett., vol. 21, no. 7, pp. 453-455, 1998.

[2] D. W. Vernooy, V. S. Ilchenko, H. Mabuchi, E. W. Streed, and H. J. Kimble, "High- $Q$ measurements of fused-silica microspheres in the near infrared," Opt. Lett., vol. 23, no. 4, pp. 247-249, 1998.

[3] V. Sandoghdar, F. Treussart, J. Hare, V. Lefevre-Segin, J.-M. Raimond, and S. Haroche, "Very low threshold whispering-gallery-mode microsphere laser," Phys. Rev. A, vol. 54, no. 3, pp. 1777-1780, 1996.
[4] F. C. Blom, D. R. van Dijk, H. J. W. M. Hoekstra, A. Sriessen, and Th. J. A. Popma, "Experimental study of integrated-optica microcavity resonators: Toward an all-optical switching device," Appl. Phys. Lett., vol. 71, no. 6, pp. 747-749, 1997.

[5] M. Cai, G. Hunziker, and K. Vahala, "Fiber-optic add/drop device based on a silica microsphere-whispering gallery mode system," IEEE Photon. Technol. Lett., vol. 11, pp. 686-687, June 1999.

[6] M. Cai, P. O. Hedekvist, and K. Vahala, "5 Gbit/s transmission through an all-fiber-optic add/drop device based on a microsphere whispering-gallery-response system," in OFC'2000 Tech. Dig., paper WM12-1, 2000.

[7] J. C. Knight, G. Cheung, F. Jacques, and T. A. Birks, "Phase-matched excitation of whispering gallery mode resonances using a fiber taper," Opt. Lett., vol. 22, no. 15, pp. 1129-1131, 1997.

[8] M. Cai and K. Vahala, "Highly efficient optical power transfer to whispering-gallery-modes by use of a symmetrical dual-coupling configuration," Opt. Lett., vol. 25, no. 4, pp. 260-262, 2000.

[9] T. E. Dimmick, G. Kakarantzas, T. A. Birks, and P. S. Russell, "Carbon dioxide laser fabrication of fused-fiber couplers and tapers," Appl. Opt., vol. 38 , no. 33, pp. 6845-6848, 1999. 